CARNETS OE Carnets de géographes

GÉOGRAPHES.

2| 2011

Espaces virtuels

\title{
Déplacements forcés et citadinités
}

Les deslocados de guerra à Maputo (Mozambique)

Jeanne Vivet

\section{(CpenEdition}

Journals

Édition électronique

URL : http://journals.openedition.org/cdg/2962

DOI : $10.4000 /$ cdg. 2962

ISSN : 2107-7266

Éditeur

UMR 245 - CESSMA

Référence électronique

Jeanne Vivet, « Déplacements forcés et citadinités », Carnets de géographes [En ligne], 2 | 2011, mis en ligne le 02 mars 2011, consulté le 07 mai 2019. URL : http://journals.openedition.org/cdg/2962 ; DOI : $10.4000 /$ cdg. 2962

Ce document a été généré automatiquement le 7 mai 2019.

\section{(c) (1)}

La revue Carnets de géographes est mise à disposition selon les termes de la Licence Creative Commons Attribution - Pas d'Utilisation Commerciale - Pas de Modification 4.0 International. 


\title{
Déplacements forcés et citadinités
}

\author{
Les deslocados de guerra à Maputo (Mozambique)
}

\author{
Jeanne Vivet
}

1 Cette thèse interroge les liens entre déplacements forcés et citadinités à partir de l'étude des déplacés de guerre d'origine rurale, venus se réfugier à Maputo, capitale du Mozambique, durant la guerre civile (1977-1992). Les principales questions posées dans ce travail sont les suivantes : Comment devient-on citadin après la fuite? Quelles citadinités émergent à l'aune des expériences violentes qui ont entraîné la déterritorialisation de ces populations?

2 L'étude de la citadinisation des deslocados à Maputo, entre géographie urbaine et géographie des migrations, s'inscrit dans une réflexion théorique sur la citadinité d'une part et sur les liens entre mobilités et territoires d'autre part, liens qui sont plus souvent analysés sous l'angle des mobilités non forcées. Des travaux issus de champs de recherche relativement cloisonnés sont ici mobilisés et articulés: les Refugee Studies et plus généralement les Forced Migration Studies et les études urbaines. Peu de travaux ont croisé la littérature sur les mobilités forcées, les migrations ville-campagne plus « classiques » et la littérature portant sur la citadinité et l'urbanisation dans les pays du Sud.

3 Sur un plan empirique, cette thèse met en perspective les mobilités forcées des déplacés par rapport à l'ensemble des mobilités de travail durablement constituées dans le sud du Mozambique et par rapport aux mobilités post-conflit, afin d'envisager les éléments de rupture, mais aussi de continuité de ces mobilités. Ce travail amène également des éléments sur les dynamiques urbaines contemporaines de Maputo, peu connues dans le monde francophone.

La déterritorialisation et la mobilité forcée constituent des expériences centrales pour comprendre les modes d'ancrage en ville des deslocados. Le caractère coercitif du déplacement vers Maputo explique que les autorités et la majorité des déplacés aient initialement envisagé leur présence en ville comme un état provisoire. La ville est avant tout le lieu du refuge et de la présence éphémère, avant de devenir pour certains, progressivement le lieu de l'installation durable, le lieu du « chez-soi ». Dans une large mesure, les deslocados se sont vus refuser le «droit à la ville » par les autorités. Maputo présentait un triple visage aux yeux des deslocados, étant à la fois le lieu du refuge, le lieu 
de l'exil et de l'altérité et enfin le lieu de l'exclusion. Les premiers temps passés en ville sont caractérisés par l'urgence; le provisoire et l'inconnu ont largement déterminé les pratiques socio-spatiales des déplacés et leurs perceptions de la vie urbaine.

Dans ce travail, la citadinisation des deslocados est comprise comme le résultat d'un processus dialectique faisant interagir les politiques des pouvoirs publics, le contexte urbain, social et familial et leurs pratiques individuelles. Si l'exceptionnalité de la situation légitime dans un premier temps leur présence, elle la rend souvent plus « illégitime » que celle des autres migrants une fois le conflit achevé.

6 Vingt ans après leur arrivée, le maintien en ville des anciens déplacés ne saurait être interprété de façon univoque ;

- pour certains, il témoigne de territorialisations positives, de leur insertion économique et résidentielle, de leur sentiment d'appartenance à Maputo ; ils sont parvenus à conforter leur place en ville et à acquérir une citadinité légitime.

- pour d'autres, le maintien en ville renvoie au contraire à un impossible retour, dans le passé et dans leur «terre d'origine » et donc à leur immobilité forcée, plutôt qu'à leur citadinité. Leur citadinité est tout à la fois fragile et marginal. Beaucoup d'entre eux subissent aujourd'hui de nouvelles mobilités forcées et sont tributaires de l'évolution foncière et économique des espaces sur lesquels ils se sont installés. Leurs citadinités entrent en résonnance directe avec certaines dynamiques urbaines post-conflit.

\section{Méthodologie}

7 La méthodologie adoptée dans cette recherche est résolument qualitative, mêlant entretiens et observations et s'appuyant également sur le dépouillement systématique de la presse du Frelimo durant les années 1980-1990 afin de recueillir les discours des autorités. Une vingtaine de chefs de quartiers ont été également interrogés.

8 Au total, 113 anciens déplacés ont été interrogés de manière approfondie ainsi que 50 autres citadins résidant dans les mêmes quartiers, jouant ainsi le rôle de groupes de contrôle et permettant de comparer leurs parcours et leurs citadinités à celle des anciens déplacés. Ces entretiens ont été effectués dans quatre quartiers de Maputo. L'entrée par quartiers a permis d'analyser les constructions territoriales des déplacés. Ces quartiers ont connu des évolutions différenciées depuis la fin des années 1980, ce qi permet d'appréhender la diversité des dynamiques urbaines post-conflit dans la capitale mozambicaine.

9 En outre, une dizaine "regressados ", c'est-à-dire des personnes ayant quitté Maputo après la fin de la guerre ont été enquêtés. Ces entretiens en zone rurale avaient un double objectif: connaître mieux les espaces dont sont originaires les déplacés de guerre et comprendre le processus de retour et ses modalités. Ces parcours de regressados se sont ainsi avérés être des contrepoints extrêmement utiles à l'étude de la citadinisation des déplacés de guerre. 
Carte 1 : Les lieux de l'enquête à Maputo.

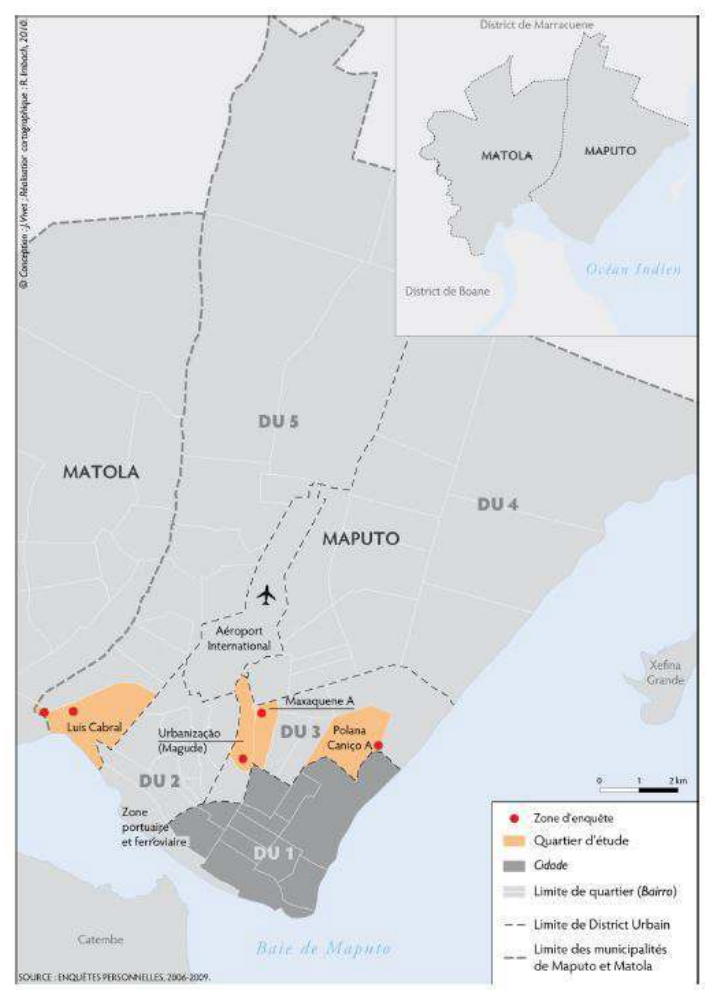

\section{Discipline \\ Géographie}

\section{Directeur}

Philippe Gervais-Lambony (Université de Paris Ouest Nanterre-La Défense)

\section{Université}

Paris Ouest Nanterre-La Défense

Membres du jury de thèse, soutenue le 26 novembre 2010

Luc Cambrézy, directeur de recherche à l'IRD

Alain Dubresson, président du jury, Université de Paris Ouest-Nanterre-La Défense Philippe Gervais-Lambony, directeur, Université de Paris Ouest-Nanterre-La Défense Elisabeth Lassailly-Jacob, rapporteur, Université de Poitiers

Isabel Raposo, rapporteur, Faculté d'Architecture de l'Université Technique de Lisbonne.

\section{Situation professionnelle actuelle}

Détachée de l'Education Nationale

Post-doc à l'Institut des Sciences Sociales de l'Université de Lisbonne (ICS) sur un projet de recherche européen financé par NORFACE.

\section{Contact de l'auteur}

jeanne.vivet@gmail.com 
INDEX

Thèmes : Carnets de soutenances 